\title{
THE DEEP-UV OPERATION OF THE OK-4/DUKE STORAGE RING FEL"
}

\author{
V.N. Litvinenko, S.H. Park, I.V. Pinayev, Y. Wu, M. Emamian, N. Hower, P. Morcombe, \\ O. Oakeley, G. Swift, P. Wang, FEL Laboratory, Physics Department, Duke University, Durham, \\ $\mathrm{NC}$
}

\begin{abstract}
In this paper we present the result of the deep-UV lasing with the OK-4/Duke storage ring FEL. The OK-4 FEL was initially commissioned in the near-UV at Duke Free Electron Laboratory in November, 1996 followed by a year-long shutdown. After re-establishing the storage ring operation in early 1998, the OK-4 FEL lasing was demonstrated in the wavelength range of 217 to $256 \mathrm{~nm}$ in 1998. A number of lasing modes with electron energies ranging from 300 to $750 \mathrm{MeV}$ have been established to accommodate application research. Starting September, 1998, the coherent deep-UV radiation is used for applications in cornea surgery, cell biology, surface physics, and nuclear physics. In this paper we report the results of the deep-UV lasing with the OK-4/Duke storage ring FEL as well as our plans to advance towards the VUV.
\end{abstract}

\section{INTRODUCTION}

The 1.1 GeV Duke storage ring was commissioned in November, 1994 [2] and later was used for number of FEL experiments. The development of the OK-4/Duke storage ring FEL started in April 1992 when the Duke FEL Laboratory and Budker Institute of Nuclear Physics (Novosibirsk, Russia) signed a Memorandum of Understanding on collaborative research in development of the XUV FELs [3]. The OK-4 FEL was previously employed for the visible and the UV lasing at VEPP-3 storage ring [4]. It was transferred to the Duke FEL laboratory (DFELL) in May of 1995 and was commissioned in November, 1996, by demonstrating lasing in the near UV and the visible [5] and production of nearly monochromatic $\gamma$-rays via Compton back-scattering [6]. At the middle of December, 1996 the operations of the Duke storage ring has been stopped and next operations of the OK-4 FEL was possible only in 1998. In April, 1998 we demonstrated lasing in the deep-UV range with tunability from $226 \mathrm{~nm}$ to $256 \mathrm{~nm}$ and also tested the operation of newly build gain modulator [7]. After three months shut-down for construction of initial stage of new Keck Life Science building, we reestablished operation of the OK-4 FEL and lased at shorter wavelength down to $217 \mathrm{~nm}$. The set of mirrors used for this lasing turned out not to be radiation resistive and mirrors degraded within two weeks to unusable condition. In September, 1998 we re-installed mirrors with central wavelength of $245 \mathrm{~nm}$, reestablished lasing and used these set-up for user program till the February, 1999 [8]. During these six months of operation we delivered OK-4 FEL beams to our user on regular basis for experiments in cornea surgery, cell biology, photo-emission electron microscopy [9] and $\gamma$ ray nuclear physics (10-40 MeV) [1].

At present, we operate the OK-4 FEL in the visible/near UV ranges (345- $730 \mathrm{~nm}$ ) to generate low energies (2 - 20 $\mathrm{MeV}$ ) monochromatic $\gamma$-rays via Compton back-scattering [1].

\section{OK-4/ DUKE STORAGE RING FEL}

The layout of the Duke storage ring with the OK-4 FEL, and the $270 \mathrm{MeV}$ linac-injector are shown on Fig.1. Most of the technical parameters of the storage ring and the OK4 FEL has been presented in our previous publications $[10,8]$. The recent developments of the OK-4/Duke storage ring FEL involved installation and commissioning closed orbit measuring system comprised of 34 units of Bergoz BPM electronics [11], the new timing system, the gain modulator [7]. We have also purchased the Hamamatsu streak camera with 2 ps resolution which is used for both storage ring and the OK-4 FEL diagnostics. These systems enhanced diagnostics capability of the OK4/Duke storage ring system and the use of closed orbit measuring system ended previously used DFELL "blindfolded technique" of the UV FEL alignment. We have improved the performance of the linac-injector by raising its energy to $270 \mathrm{MeV}$ and by installing the new nitrogen laser with higher power and low jitter (less than $1 \mathrm{nsec}$ ) to drive photo-cathode for single bunch injection. At present, the system is capable of continuos injection with 0.5 $\mathrm{mA} / \mathrm{shot}$ rate in the desirable RF bucket with no spills. Maximum stored current in a single bunch is limited to 5 - $15 \mathrm{~mA}$. Above the threshold value, electron beam develops vertical instability and reduces its intensity by about a half. This threshold value does not exhibit strong dependence on the global parameters such as chromaticity or betatron tunes but depends on the beam orbit and HOMs in the RF cavity. The nature of the effect limiting the current per bunch is not obvious and we study it in order to improve this parameter in the future. A typical set of Duke storage ring parameters used for described experiments is listed in the Table I.

\footnotetext{
* Work is supported ONR grant \#N00014-941--0818
} 
DUKE FEL STORAGE RING

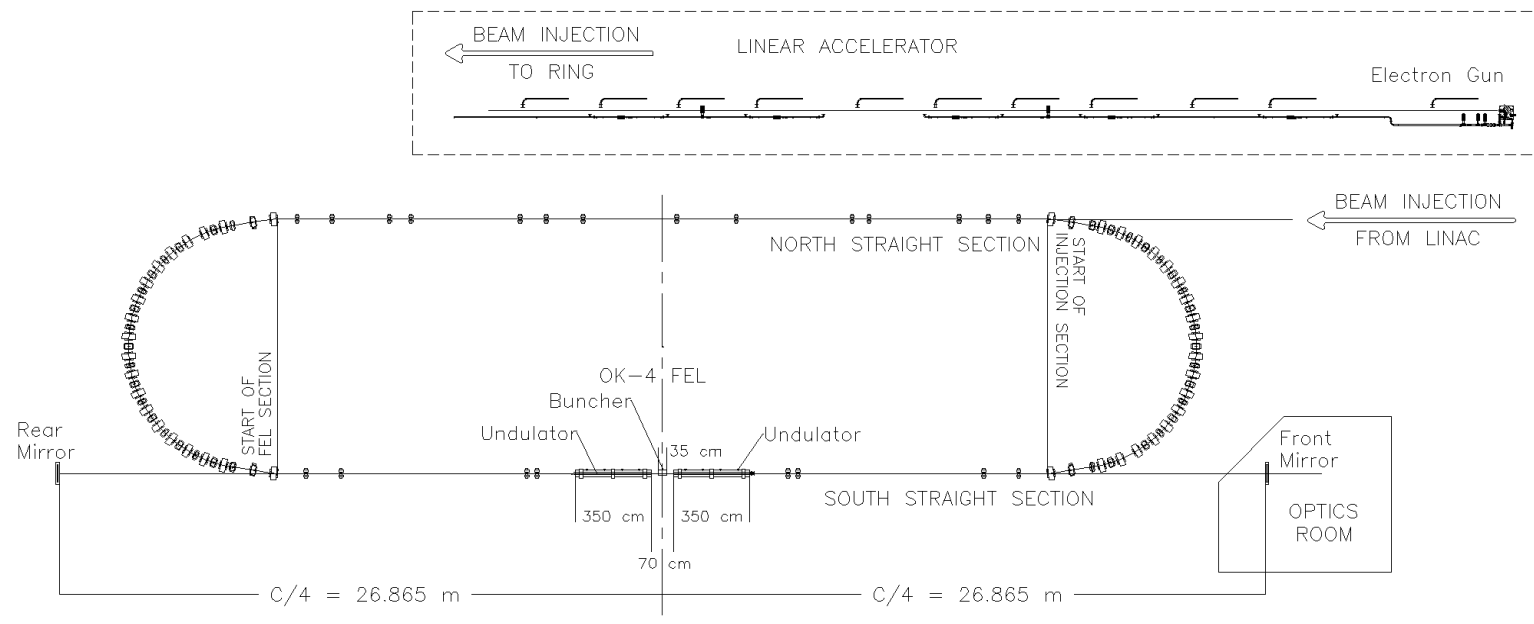

Figure 1. Layout of the Duke/OK-4 storage ring FEL.

Table 1. Typical parameters used for the deep-UV lasing

\begin{tabular}{ll}
\hline Electron Energy [GeV] & $0.3-0.75$ \\
RF voltage (178 MHz), kV & $200-550$ \\
Number of electron bunches & $1-3$ \\
Beam current [mA], per bunch & $3-8$ \\
Broad band impedance, $\mathrm{Zn} / \mathrm{n}[\mathrm{Ohm}]$ & $3.25 \pm 0.25$ \\
Typical lifetime with laser on [hours] & $1-3$ \\
\hline
\end{tabular}

The only improvement in the OK-4 FEL system was the increase of currents in both power supplies for the electromagnetic wigglers (to $2.7 \mathrm{kA}$ and maximum $\mathrm{K}_{\mathrm{w}}$ to 4.6) and in the buncher (to $1.5 \mathrm{kA}$ ). These up-grades allowed us to operate at higher energies of electron beam (up to $750 \mathrm{MeV}$ ) and to generate higher average power.

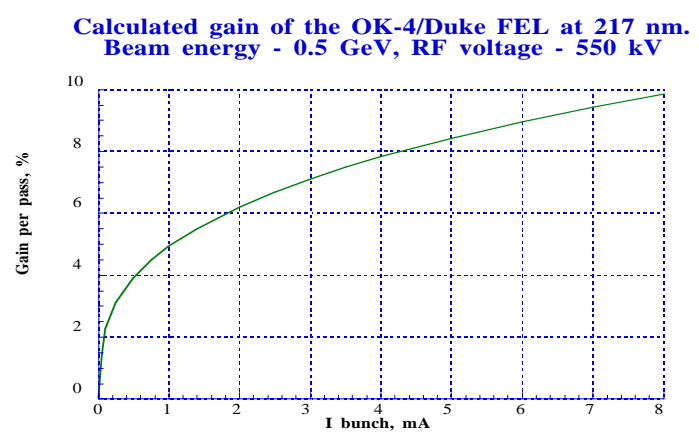

Fig.2. Typical calculated dependence of the OK-4/Duke FEL gain on the electron current per bunch. We used broad-band impedance of $\mathrm{Z}_{\mathrm{n}} / \mathrm{n}=3.25 \mathrm{Ohm}$ for this calculations.

The $53.73 \mathrm{~m}$ long optical cavity of the OK-4 FEL comprises of two mirrors with $26.46 \mathrm{~m}$ radii and has Rayleigh range only $3 \mathrm{~m}$. It makes is very susceptible to vibrations and angular errors. The design of the optical cavity and a its sophisticated feed-back and control system are described elsewhere [5, 12].

The present vacuum chambers of Duke storage ring have very large longitudinal impedance (see Table 1) which causes microwave instability to start at sub-mA currents per bunch. Well-developed and saturated microwave instability determines the energy spread and bunchlengthening of the beam at most of operating parameters listed in Table. 1, and therefore determines the maximum gain attainable with the OK-4 FEL. Fig. 2 shows dependence of the maximum gain in the OK-4/Duke FEL for deep-UV with typical cubic root dependence on current per bunch. Our direct measurements of the maximum gain are in good agreement with our predictions.

\section{LASING IN THE DEEP-UV}

The deep-UV lasing with the OK-4/Duke storage ring FEL has been demonstrated in April and August of 1998 using a number of set-ups and energies. Generally, lasing was easier to achieve and was more stable when we operate storage ring at higher energies (i.e. from 500 to 750 $\mathrm{MeV})$. We contribute this effect to better stability of electron beam and power supplies.

We used two sets of multilayer dielectric mirrors custom manufactured by Lumonix Optics Group (Canada). The reflectivity bands of these mirrors as well as typical tunability ranges attained during initial lasing runs are shown on Fig.3.

Mirrors with central wavelength of $225 \mathrm{~nm}$ were not radiation resistive and we did not use them for user applications. Starting September, 1998 we used the 245 $\mathrm{nm}$ mirrors to provide the OK-4 FEL laser beams to our users on regular scheduled basis for six months in both $\mathrm{CW}$ and pulsed mode. We out-coupled maximum of 100 $\mathrm{mW}$ of average laser power per mirror into TEMoo mode using $700 \mathrm{MeV}$ electron beam with $16 \mathrm{~mA}$ average current in two bunches. Giant pulses with 50-200 microsecond duration were generated using the gain modulator [7], at a maximum reprate of $30 \mathrm{~Hz}$ with maximum $0.25-0.4 \mathrm{~mJ}$ per macropulse and a peak out-coupled power of about 0.3 MW. In addition to the full transverse coherence (TEMoo mode) of the OK-4 FEL radiation we demonstrated full 
longitudinal coherence by generating the Fourier limited Gaussian wavepackets with RMS duration close to 2 ps and RMS linewidth close to $0.003 \%$.
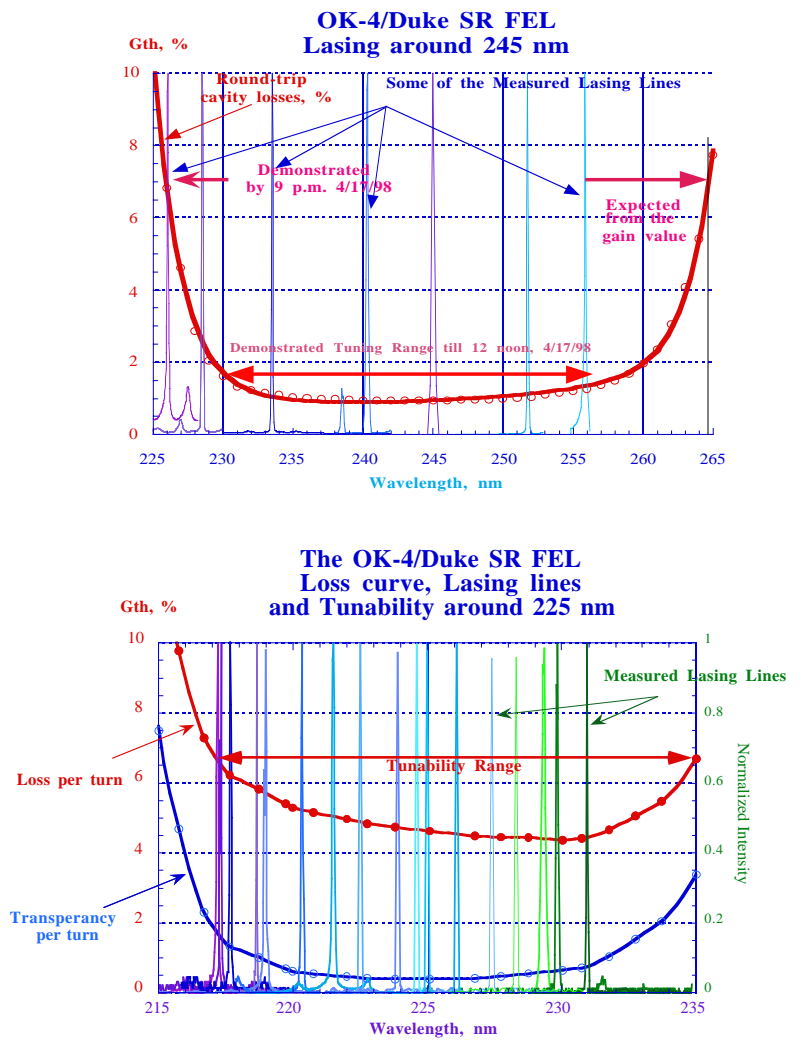

Fig.3 Tunability of the OK-4/Duke FEL operating at $500 \mathrm{MeV}$ around $245 \mathrm{~nm}$ (April 3, 1998: $1.98 \mathrm{~mA} /$ bunch, $500 \mathrm{MeV}$, $200 \mathrm{kV} \mathrm{RF}$ ) and around $225 \mathrm{~nm}$ (August 11, 1998: 1.5 $\mathrm{mA} /$ bunch, $500 \mathrm{kV} \mathrm{RF}$ ). The thick-solid curve shows measured round trip losses and thin curves show samples of measured spectra. The stop-bands define the level of the FEL gain. From the above graph we concluded that the OK-4 FEL has the gain at least 6.9\% per pass@ $226 \mathrm{~nm}$ and 6.5\% per pass@ @217 $\mathrm{nm}$ with the above beam parameters.

\section{CONCLUSIONS}

The OK-4/Duke storage ring FEL has demonstrated reliable lasing as well as generated usable laser beams in the deep-UV range of spectrum. The OK-4 FEL demonstrated average spectral brightness of $(2-4) \cdot 10^{20}$ and peak spectral brightness of $3 \cdot 10^{26}$ $\mathrm{ph} / \mathrm{sec} / \mathrm{mm}^{2} / \mathrm{mrad}^{2} /\left(10^{3} \cdot \mathrm{BW}\right)$ in the deep-UV spectral range. The transparency of used mirrors was too low for the optimized out-coupling. At the central wavelength of 245 $\mathrm{nm}$, the mirror transparency of $0.035 \%$ and initial loss of $0.47 \%$ provide an outcoupling efficiency less than $8 \%$. We ordered a new set of mirrors with transparency of 0.5 $0.75 \%$ at the central wavelength for better extraction efficiency. These modifications as well as extension of the OK-4 FEL operation below $200 \mathrm{~nm}$ will enhance capability and strength of our user program. The UV and $\gamma$-ray beams from the OK-4 FEL will be soon delivered into newly built Keck Life Science Laboratory housing the FEL users. The exploration of the shorter wavelength range with the OK-4/Duke storage ring FEL will continue in parallel with the user applications. We are in the process of design and construction of the $22 \mathrm{~m}$ long OK-5 FEL comprising four electromagnetic helical wigglers with switchable polarization. The OK-5 FEL will have gain in excess of $100 \%$ per pass and will allow us to lase in the VUV range.

\section{ACKNOWLEDGMENTS}

Authors are thankful to the staff of the Duke University Free Electron Laboratory, to our collaborators from Budker Institute of Nuclear Physics and A. Lumpkin from Argonne National laboratory for their important contributions to the success of the OK-4/Duke storage ring FEL. Authors would like to acknowledge the Office of Naval Research for its continuous support of the Duke OK-4 UV FEL and its applications.

\section{REFERENCES}

[1] S.H.Park et al., "Performance of the Duke storage ring $\gamma$-ray source", Presented at these Particle Accelerator Conference

[2] V.N.Litvinenko et al., Proc. of PAC'95, 1995, p. 213.

[3] V.N.Litvinenko, J.M.J.Madey, N.A.Vinokurov, Proc. of PAC' 93, 1993, p. 1442

[4] V.N.Litvinenko, "The optical klystron on VEPP-3 storage ring lasing in the visible and the ultraviolet", Thesis, Novosibirsk, 1989; G.N.Kulipanov et al., NIM A296 (1990) 1;

[5] V.N.Litvinenko et al., NIM, A 407 (1998) 8 V.N.Litvinenko et al., Proc. of PAC'97, 1997, P.883

[6] V.N.Litvinenko et al., Phys.Rev. Lett., V.78, N.4, June 1997, p. 4569

[7] I.V.Pinayev et al., "Critical Systems for High Peak Power Storage Ring FEL", these proceedings.

[8] Y. Wu et al., NIM B 144 (1998) 90

[9] H.Ade et al., Surface Review and Letters, Vol. 5, No. 6 (1998) 1257

[10] Y.Wu, V.N.Litvinenko, B.Burnham, S.H.Park and J.M.J.Madey, p. 1753 IEEE Transaction on Nuclear Science, Vol. 44, No. 5, 1997,

V.N.Litvinenko et al., "The Duke XUV FEL Storage Ring

Facility", Proc. of the first APAC Conference, March, 1998, Tsukuba, Japan.

V.N.Litvinenko et al., NIM A375 (1996) 46-52

[11] P.Wang et al., "Beam Position Monitors for Duke FEL Storage Ring", these proceedings.

[12] I.V.Pinayev et al., AIP Proc., CP451 (1998) 545 